Is there an independent association between burnout and increased allostatic load? Testing the contribution of psychological distress and depression

Hintsa, Taina

2016-08

Hintsa , T , Elovainio , M , Jokela, M , Ahola , K, Virtanen , M \& Pirkola , S 2016 , ' Is there an independent association between burnout and increased allostatic load? Testing the contribution of psychological distress and depression ' , Journal of Health Psychology , vol. 21 , no. 8 , pp. 1576-1586 . https://doi.org/10.1177/1359105314559619

http://hdl.handle.net/10138/224473

https://doi.org/10.1177/1359105314559619

publishedVersion

Downloaded from Helda, University of Helsinki institutional repository.

This is an electronic reprint of the original article.

This reprint may differ from the original in pagination and typographic detail.

Please cite the original version. 


\title{
Is there an independent association between burnout and increased allostatic load? Testing the contribution of psychological distress and depression
}

Journal of Health Psychology 2016, Vol. 21 (8) 1576-1586 (c) The Author(s) 2014

Reprints and permissions: sagepub.co.uk/journalsPermissions.nav DOI: $10.1177 / 1359105314559619$ hpq.sagepub.com

@SAGE

\author{
Taina Hintsa', Marko Elovainio², Markus Jokela', \\ Kirsi Ahola ${ }^{3}$, Marianna Virtanen ${ }^{3}$ and \\ Sami Pirkola 2,4,5,6,7
}

\begin{abstract}
Burnout has been suggested to be related to depression. We examined the relationship between burnout and allostatic load, and whether this association is independent of psychological distress and depression. We measured burnout psychological distress, depression, and allostatic load in 3283 participants. Higher burnout $(\beta=0.06, p=0.003)$ and cynicism $(\beta=0.03, p=0.03 \mathrm{I})$ and decreased professional efficacy $(\beta=0.03, p=0.007)$ were related to higher allostatic load independent of age, sex, education, occupation and psychological distress. Depression, however, explained 60 percent of the association. Burnout is related to higher allostatic load, and this association partly overlaps with co-occurring depression.
\end{abstract}

\section{Keywords}

allostatic load, burnout, depression, psychological distress

\section{Introduction}

Burnout has been defined as a negative workrelated state of mind that is preceded by chronic work stress (Schaufeli and Enzmann, 1998; Shirom, 2003), and it is assumed to be caused by an imbalance between the employee and the working conditions. The original concept of burnout consists of three dimensions: exhaustion, cynicism, and lack of professional efficacy (Maslach et al., 2001; Schaufeli et al., 1996). Exhaustion refers to the feeling of not being able to offer any more of oneself at an emotional level; cynicism denotes a distant attitude toward work,

\footnotetext{
'University of Helsinki, Finland

${ }^{2}$ National Institute for Health and Welfare, Finland

${ }^{3}$ Finnish Institute of Occupational Health, Finland

${ }^{4}$ Lapland Hospital District, Finland

5 University of Oulu, Finland

${ }^{6}$ Helsinki University Central Hospital, Finland

7University of Tampere, Finland
}

\section{Corresponding author:}

Taina Hintsa, Department of Personality, Work and Health Psychology, Institute of Behavioural Sciences, University of Helsinki, P.O. Box 9, Helsinki Fl-000 14, Finland.

Email: taina.hintsa@helsinki.fi 
employees, and colleagues; and lack of professional efficacy reflects the feeling of not performing tasks adequately well or being incompetent at work (Maslach et al., 1996, 2001). Burnout is coded in International Classification of Diseases, 10th Revision (ICD-10) (World Health Organization, 1992) as a condition that influences health (Honkonen et al., 2006; Shirom and Melamed, 2005; Toker et al., 2012).

As burnout is a stress-induced state, the physiological stress system is involved, that is, autonomous nervous system (ANS) and the hypothalamic-pituitary-adrenal axis (HPA axis). Real or perceived threat activates the sympathetic-adrenal-medullary (SAM) axis to release catecholamines and the HPA axis to secrete glucocorticoids (Sapolsky et al., 2000). Elevated levels of these stress hormones strain many biological systems in the body. Allostasis, the process through which organisms adapt to environments through the activation of neural, neuroendocrine, and neuroendocrine-immune mechanisms, refers to short-term adaptation. Allostatic load (AL), in turn, represents physiological strain of an organism, that is, long-term maladaptation (McEwen and Stellar, 1993). It is a result of the organism's inability to shut off allostatic activity after stress, that is, a prolonged or repeated activation of SAM and HPA axes due to stressful situations. Allostatic mediators function as contributors to the development of $\mathrm{AL}$ and include overactivation of primary stress mediators and effects; dysregulation of cardiovascular, metabolic, and inflammatory systems; and outcomes such as cardiovascular disease, depression, and mortality (McEwen, 1998).

Prior evidence has shown an association between burnout and increased AL among 104 female school teachers (Bellingrath et al., 2009) and 30 healthy men and women of different occupations (Juster et al., 2011a). Burnout has been related to secondary mediators of AL (immune alterations) such as flu-like infections and common cold (Mohren et al., 2003). Burnout has also been shown to relate to changes in monocyte anti-inflammatory immune function (Mommersteeg et al., 2006).
The opposite findings also exist. It was found among 338 Dutch managers that AL was not related to burnout (Langelaan et al., 2007). A recent systematic review of biomarkers of burnout concluded that there were no significant biomarkers of burnout (Danhof-Pont et al., 2011). They suggested that this may be largely due to the incomparability of studies due to their heterogeneity, differences in assessment of burnout, and sample sizes (Danhof-Pont et al., 2011). The contribution of psychological distress and depression on the associations between burnout, its components, and AL has not previously been reported.

The question of whether there is any independent effect of burnout on health outcomes arose from the findings showing its rather strong correlation with psychological distress and depressive disorders (McEwen, 2003). Addressing the question about whether burnout would have an independent status as a condition that affects health irrespective of comorbidities such as mood disorders may be difficult solely by the means of self-reported symptoms. Burnout has been suggested to be related to depression especially in the work context (Warr, 1987). Burnout and depression have been differentiated statistically among Dutch teachers (Bakker et al., 2000). The development of burnout might be related to a lack of reciprocity in the occupational domain, whereas lack of reciprocity in intimate relationships was related to depressive symptoms (Bakker et al., 2000). It has been also reported that burnout and depression complement each other and cover partly overlapping phenomena in a population-based sample (Ahola et al., 2005). Only half of the severely burned-out employees, however, were clinically depressed in the Health 2000 study (Ahola et al., 2005). Nevertheless, the overlap between burnout and depression has been previously shown for eight of the nine major depressive episode diagnostic criteria of the Diagnostic and Statistical Manual of Mental Disorders (Bianchi et al., 2013). It is possible that co-occurring depression or symptoms of psychological distress account for the association between burnout and AL. In this study, we 
examined whether burnout is related to $\mathrm{AL}$ independent of psychological distress and depression in a large population-based sample.

\section{Materials and methods}

\section{Participants and design}

A multidisciplinary epidemiologic Health 2000 Study was conducted in 2000-2001 in Finland (Aromaa and Koskinen, 2004). The sample is representative of the Finnish population and included 8028 persons aged 30 years or over. The details of the sample and the sampling procedure have been described in detail previously (Ahola et al., 2006a; Aromaa and Koskinen, 2004). There were 5956 participants who were aged 65 years or less. Of those, 3283 were currently employed and had full information on all relevant study variables.

\section{Measures}

Burnout was measured with the Maslach Burnout Inventory-General Survey (MBI-GS; (Schaufeli et al., 1996) which consists of 16 items which form three subscales: emotional exhaustion ( 5 items, Cronbach's $\alpha=0.91$ ), cynicism ( 5 items, $\alpha=0.79$ ), and lack of professional efficacy ( 6 reversed items, $\alpha=0.82$ ) (Ahola et al., 2006b). The responses were given on a 7 -point scale $(0=$ never and $6=$ daily $)$. A burnout score was calculated, weighing each dimension so that the scores corresponded to the original response scale $(0.4 \times$ exhaustion $+0.3 \times$ cynicism $+0.3 \times$ lack of professional efficacy) as suggested by previous studies (Ahola and Hakanen, 2007; Kalimo et al., 2006).

Psychological distress was assessed by the 12-item General Health Questionnaire (GHQ12) which is a self-report instrument for assessing psychological distress (Goldberg, 1972; Goldberg and Williams, 1978). The psychometric properties of GHQ-12 have been reported to be good (McCabe et al., 1996; Schmitz et al., 1999) and sensitive in screening common mental disorders (Goldberg et al., 1997), such as affective disorders (symptoms of anxiety and depression), social dysfunction, and loss of self-confidence (Mäkikangas et al., 2006; Penninkilampi-Kerola et al., 2006).

Depression was self-rated with the original Beck Depression Inventory which consists of 21 items with four alternative statements (from 0 to 3) for each item (Beck et al., 1988). As done previously in a Finnish study (Ahola et al., 2006b), a linear score of depression was calculated. Beck Depression Inventory has been used in epidemiological studies (Nabi et al., 2010), health behavior interventions (Karapolat et al., 2009), and studies on effectiveness of psychotherapy (Knekt et al., 2008). sychometric properties of Beck Depression Inventoryhave been reported to be good in several studies (Beck et al., 1988), and there is evidence on the validity of it (Aben et al., 2002; Aikens et al., 1999; Nuevo et al., 2009).

The AL was operationalized as an index that was based on biological parameters that have been previously used and have shown to represent the wear and tear of the body (Juster et al., 2010; Seeman et al., 1997). The following biomarkers (cardiovascular, metabolic, inflammation, and anthropometric) were measured during a clinical health examination: systolic and diastolic blood pressure; cholesterol (highdensity lipoprotein (HDL) cholesterol, lowdensity lipoprotein (LDL) cholesterol, and total cholesterol), triglycerides, glucose, insulin, and glycosylated hemoglobin (HbA1c) in plasma; homocysteine and C-reactive protein (CRP); and body mass index (BMI) and waist circumference. Each of the biomarkers was coded in no-risk (=0) and high-risk groups (=1) according to adapted national clinical cut points when available, and then an index of AL was formed by summing up these indicators (range: 0-13).

In the analyses, covariates were age, sex, completed years of education, and occupation (manual, lower non-manual, upper nonmanual, and entrepreneur). In addition, psychological distress and depression were controlled for.

\section{Statistical analyses}

First, the correlations between study variables were calculated. Then, the association between 
Table I. Descriptive characteristics of the sample.

\begin{tabular}{llll}
\hline Demographics & Mean (\%) & SD & Range \\
\hline Age & 44.40 & 8.44 & $30-65$ \\
Gender & & & \\
$\quad$ Male & $1629(49.6)$ & & \\
$\quad$ Female & $1654(50.4)$ & & $0-33$ \\
Education (years) & 12.93 & 3.59 & \\
Occupation & & & \\
$\quad$ Manual & $963(29.3)$ & & \\
Lower non-manual & $917(27.9)$ & & \\
$\quad$ Upper non-manual & $898(27.4)$ & & $0-5.6$ \\
$\quad$ Entrepreneur & $505(15.4)$ & & $0-6$ \\
Burnout & 1.13 & 0.90 & $0-6$ \\
$\quad$ Exhaustion & 1.10 & 1.14 & $0-6$ \\
$\quad$ Cynicism & 1.08 & 1.16 & \\
$\quad$ Lack of professional efficacy & 1.24 & 1.18 & $0-12$ \\
Mood disorder & & & $0-41$ \\
$\quad$ Psychological distress & 1.58 & 2.58 & $0-13$ \\
$\quad$ Depression & 5.40 & 5.82 & $0-3.46$ \\
Allostatic load & 2.24 & 2.32 & 0.91 \\
Allostatic load (square root transformation) & 1.18 & & \\
\hline
\end{tabular}

SD: standard deviation.

burnout and AL was tested using linear regression analyses with continuous AL index as the outcome. The distribution of AL was skewed, and therefore, a square root transformed variable was formed and used in the analyses. $B$ coefficients were expressed as standardized coefficients. The analyses were sequentially adjusted for first age and sex, then education and occupation, and finally for psychological distress and depression. We used weighting adjustment and sampling parameters in the analyses to correct possible bias caused by the complex sample survey data and the loss of participants (Heistaro, 2008). We used PASW 18.0 and STATA 13 for statistical analyses.

\section{Results}

The descriptive statistics of the sample are shown in Table 1 and the frequencies of AL biomarkers in high-risk and no-risk groups in Table 2.

Burnout correlated with psychological distress, $r=0.51(p<0.001)$, and depression, $r=0.57(p<0.001)$. The correlation between psychological distress and depression was $r=0.66(p<0.001)$.

The results of linear regression analyses showed that burnout was associated with $\mathrm{AL}$ $(B=0.08, p<0.001)$. The association was found to be independent of age, sex, education, occupation type, and psychological distress $(B=0.06$, $p=0.003$ ) (Table 3 ). When depression was taken into account in the analyses, burnout was no longer related to AL (Model 5, $\mathrm{p}=0.272$ ). We found that depression accounted for 60 percent of the association between burnout and $\mathrm{AL}$ (from $B=0.05$ to $B=0.02$ ). Of the components of burnout, cynicism $(B=0.05, p=0.001)$ and lack of professional efficacy $(B=0.07, p<0.001)$ were associated with AL (Table 3 ). These associations were found to be independent of age, sex, education, occupation type, and psychological distress: cynicism $(B=0.03, p=0.031)$ and lack of professional efficacy $(B=0.03, p=0.007)$ (Table 3). Taking depression into account, the associations were no longer significant. Depression accounted for 66 percent of the association between cynicism and AL and 50 percent 
Table 2. Biomarkers of AL.

\begin{tabular}{|c|c|c|c|}
\hline \multirow[t]{2}{*}{ Biomarkers of AL (units) } & \multirow{2}{*}{$\frac{N(\%)}{\text { No-risk }(=0)}$} & \multirow{2}{*}{$\frac{N(\%)}{\text { High-risk }(=1)}$} & \multirow[t]{2}{*}{ Cut-off point } \\
\hline & & & \\
\hline \multicolumn{4}{|l|}{ Cardiovascular } \\
\hline Systolic blood pressure $(\mathrm{mmHg})$ & $2439(74.3)$ & $844(25.7)$ & 140 \\
\hline Diastolic blood pressure $(\mathrm{mmHg})$ & $2416(78.6)$ & $867(26.4)$ & 90 \\
\hline \multicolumn{4}{|l|}{ Metabolic } \\
\hline Total cholesterol (mmol/L) & $2523(76.9)$ & $760(23.1)$ & 6.5 \\
\hline High-density lipoprotein cholesterol (mmol/L) & $3026(92.2)$ & $257(7.8)$ & 0.9 \\
\hline Low-density lipoprotein cholesterol (mmol/L) & $2956(90.0)$ & $327(10.0)$ & 5.0 \\
\hline Triglycerides (mmol/L) & $2540(77.4)$ & $743(22.6)$ & 1.8 \\
\hline Glucose $(\mathrm{mmol} / \mathrm{L})$ & 3009 (91.7) & $274(8.3)$ & 6.0 \\
\hline Insulin (mU/L) & $2634(80.2)$ & $649(19.8)$ & 11.0 \\
\hline Glycosylated hemoglobin (\%) & $2730(83.2)$ & $553(16.8)$ & 7.0 \\
\hline \multicolumn{4}{|l|}{ Inflammation } \\
\hline Homocysteine $(\mu \mathrm{mol} / \mathrm{L})$ & $2950(89.9)$ & $333(10.1)$ & 14.3 \\
\hline C-reactive protein $(\mathrm{mg} / \mathrm{L})$ & $2874(87.5)$ & $409(12.5)$ & 3.0 \\
\hline \multicolumn{4}{|l|}{ Anthropometric } \\
\hline Body mass index $\left(\mathrm{kg} / \mathrm{m}^{2}\right)$ & $2677(81.5)$ & $606(18.5)$ & 30 \\
\hline Waist circumference $(\mathrm{cm})$ & $2567(78.2)$ & $716(21.8)$ & 100 \\
\hline
\end{tabular}

AL: allostatic load.

Table 3. The association of burnout and its components with allostatic load ${ }^{a}(n=3283)$.

\begin{tabular}{|c|c|c|c|c|c|c|c|c|}
\hline & \multicolumn{2}{|c|}{ Burnout } & \multicolumn{2}{|c|}{ Exhaustion } & \multicolumn{2}{|c|}{ Cynicism } & \multicolumn{2}{|c|}{$\begin{array}{l}\text { Lack of professional } \\
\text { efficacy }\end{array}$} \\
\hline & B & $p$-value & B & $p$-value & B & $p$-value & B & $p$-value \\
\hline Model I & 0.08 & $<0.001$ & 0.02 & 0.152 & 0.05 & 0.001 & 0.07 & $<0.001$ \\
\hline Model 2 & 0.05 & 0.002 & 0.02 & 0.100 & 0.03 & 0.039 & 0.04 & 0.001 \\
\hline Model 3 & 0.05 & 0.004 & 0.02 & 0.076 & 0.03 & 0.031 & 0.03 & 0.005 \\
\hline Model 4 & 0.06 & 0.003 & 0.03 & 0.071 & 0.03 & 0.031 & 0.03 & 0.007 \\
\hline Model 5 & 0.02 & 0.272 & 0.01 & 0.995 & 0.01 & 0.593 & 0.02 & 0.056 \\
\hline
\end{tabular}

GHQ: General Health Questionnaire; BDI: Beck Depression Inventory.

Model I: no control variables; Model 2: age and sex; Model 3: age, sex, education, and occupation; Model 4: Model 3+Psychological distress (GHQ); Model 5: Model 3+Depression (BDI).

aSquare root transformed allostatic load variable.

of the lack of professional efficacy-AL relationship. Exhaustion was not related to $\mathrm{AL}$ $(\mathrm{p}=0.152)$.

As burnout was not related to $\mathrm{AL}$ when depression was taken into account, we wanted to check using linear regression analysis whether single biomarkers of AL are related to burnout independent of all control variables including depression. None of biomarkers of AL were related to total burnout (all $p$-values $>0.05$ ). Regarding the components of burnout, lower level of insulin was related to cynicism $(p$-value $=0.014)$ and higher level of diastolic blood pressure was related to decreased professional efficacy $(p$-value $=0.019)$ and independent of all control variables. 


\section{Discussion}

In this study of 3283 Finnish employees from various occupations in a nationally representative sample, we examined whether burnout is associated with AL and whether this association is independent of psychological distress and depression. After adjustment for covariates (age, sex, education, occupation), burnout was associated with higher AL independent of psychological distress. However, the association between burnout and AL was to a great extent accounted for by depression; thus, the question of whether burnout has an independent physiological effect, as expressed by increased AL, remains unanswered.

We found that burnout was associated with higher AL. This finding is in line with prior small-scale studies: one study reported increased level of AL to be associated with the frequency of total burnout symptoms (Juster et al., 2011a). In another study, chronic work stress and exhaustion were related to higher AL in female school teachers (Bellingrath et al., 2009). However, a study among male managers employed in a Dutch Telecom Company reported that AL focused on secondary outcomes was not higher among burned-out male managers compared to healthy controls (Langelaan et al., 2007). In addition to occupational differences, these divergent results might be explained by differences in the measurement of AL and sample sizes. All in all, a systematic review suggested that the prior inconsistencies between several studies of biomarkers in burnout are largely due to the heterogeneity-related incomparability of the studies (Danhof-Pont et al., 2011). In addition, the role of psychological distress or depression in the association between burnout and AL has not previously reported.

In this study, the observed association between burnout and increased AL was not independent of depression, as depression explained 60 percent of the association. This is in line with a previous study reporting that burnout and major depressive episode are associated with similar amount of depressive symptoms (Bianchi et al., 2013). AL is suggested to result from continuous overactivation of stress hormones and cellular activity and to lead to dysregulation of cardiovascular, metabolic, and inflammatory systems and tertiary outcomes such as cardiovascular disease, depression, and mortality (McEwen, 1998; McEwen and Stellar, 1993). In depression, elevated levels of stress hormones, dysregulation of the ANS (Thayer et al., 1998) and HPA axis activity, and hyperactivation of the amygdala (Sheline et al., 2001) have been demonstrated, all of which may be related to overactivation of systems involved in physiological stress response (McEwen, 2003).

The association between burnout and depression has been shown to be reciprocal (Ahola and Hakanen, 2007). Burned-out employees may be more vulnerable to depression and depressed employees to burnout. The path from burnout to depression appears, however, to be stronger than the path from depression to burnout (Ahola and Hakanen, 2007). Therefore, the risk of having depression may rise along with the increasing level of burnout (Ahola et al., 2005). Indeed, a full-panel three-wave longitudinal study among a large sample of employees showed that increasing burnout predicts increasing depression and vice versa (Toker and Biron, 2012). Our finding on the independent effect of burnout on AL after adjustment for psychological distress but not after adjustment for depression suggests that the association between burnout and AL represents their overlap with depression. This may be caused by effect mediation; when burnout becomes more severe, it is more likely to be associated with depression, which in turn strengthens AL. It is also possible, however, that $\mathrm{AL}$ is an underlying cause for both burnout and depression. Prospective studies are needed to test this hypothesis.

In their review of biomarkers of burnout, Danhof-Pont et al. (2011) emphasized the need to investigate the contribution of components of burnout (Danhof-Pont et al., 2011). Schnorpfeil et al. (2003) were among the first to apply the AL concept to psychosocial work characteristics (Schnorpfeil et al., 2003). Their study revealed a weak but significant association between an extended AL score based on 14 
parameters and job-demands in industrial workers. Our results showed that emotional exhaustion was not related to AL. This is not in line with previous studies reporting that higher AL is associated with higher level of emotional exhaustion (Bellingrath et al., 2009; Juster et al., 2011a). So far, information on biomarkers of the components of burnout is scant, and thus, more research is needed.

We found that of the components of burnout, cynicism and the lack of professional efficacy were associated with AL independently of psychological distress. The associations may partly be explained by negative emotions caused by chronic stress and behaviors related to them (Ranjit et al., 2007). Cynical distrust has previously been associated with interleukin-6 (IL-6) and CRP (Ranjit et al., 2007; Steptoe et al., 2008). Chronic stress has been found to be associated with inflammatory biomarkers, and higher levels of cynical distrust were associated with progressively higher levels of the inflammatory markers (Ranjit et al., 2007). Cynicism is likely to also increase interpersonal conflicts and related difficulties at work (GarciaIzquierdo and Rios-Risquez, 2012) and through that may alter the functions of physiological stress system.

Lack of professional efficacy reflects decreased ability to perform well at work and perceptions of incompetence. It could either increase the efforts at work or, depending on successfulness of these efforts, depressed mood due to feelings of incompetence, both of which could compromise the functions of the physiological stress system. Impaired cognitive performance in memory and attention in patients with chronic burnout syndrome have been reported (Sandström et al., 2005). They suggest that cognitive impairment could be due to dysfunction of HPA axis (Cintra et al., 1994). A persistent lack of cortisol availability in chronically stressed individuals may promote an increased vulnerability for the development of stress-related bodily disorders (Heim et al., 2000).

Decreased cortisol response has been associated with depressed mood among those who have low self-esteem (Scarpa and Luscher, 2002). Reduced cortisol response in participants with lower levels of self-esteem may be related to unsuccessful coping in reaction to a difficult task (Scarpa and Luscher, 2002). Lack of professional efficacy is likely to influence self-esteem and involve pessimistic beliefs and feelings of hopelessness. It has been suggested that self-esteem affects the endocrine stress response, that is, it may play a significant role in the regulation of the HPA axis (Pruessner et al., 1999). Low self-esteem of subjects who believe to perform poorly on a given task is associated with HPA axis response (Pruessner et al., 1999). Studies on biomarkers related to pessimism (the general tendency to expect negative outcomes) have shown that pessimism is associated with higher levels of inflammation independent of depression in a healthy population (Roy et al., 2010) and increased stimulation of the HPA axis (Das and O'Keefe, 2006), which could contribute to chronic inflammation. Pessimistic attributions have also been associated with vulnerability to hypertension (Grewen et al., 2000) and hopelessness with incident hypertension in Finnish men (Everson et al., 2000).

The main limitation of this study is that the design is cross-sectional and thus does not allow conclusions about causalities. Although we showed an association between burnout and AL stemming from lack of professional efficacy and cynicism independently of psychological distress, a cross-sectional study does not allow conclusions about causalities. So, it is possible that a decrease in professional efficacy precedes an increase in AL or vice versa. We did not find that burnout exerts a physiological effect independently of depression. As psychological stress, however, may be a precursor of burnout and depression, a longitudinal study could help to further clarify the relationship between psychological distress, depression, burnout, and AL. Another limitation is the operationalization of AL as it did not include biological biomarkers of stress equally across all 
categories, and measures of cortisol and other stress hormones were not available. An adequate amount of parameters, however, were available in the data to achieve a proxy measure of AL. Furthermore, burnout, psychological distress, and depression were self-rated, which may make the results vulnerable to common method variance and bias due to response style. Burnout was measured with the most commonly used instrument to measure burnout, that is, the MBI-GS, psychological distress with GHI, and depression with BDI. These have been found to be valid scales for screening and research purposes (Aalto et al., 2012; Schutte et al., 2000; Taris et al., 1999). As we had a population-based sample, these findings may not apply to more severely affected subjects fulfilling the diagnostic criteria of depression and/or clinical burnout.

The AL index, however, was formed from objectively measured biomarkers, which is a major strength of this study. This study was the first one with a large sample size which in addition was population-based and representative of a wide variety of jobs and occupations. This increases the generalizability of the present findings. A large sample also enabled us to study the association between burnout and AL independently of age, gender, education, and occupation.

In conclusion, burnout is associated with higher AL independently of socio-demographic factors and psychological distress. However, the causality between burnout and $\mathrm{AL}$ and whether the association is independent of co-occurring depression remain to be investigated. Although our population-based study does not allow direct clinical implications, it may indirectly support the screening of psychiatric symptoms and use of simple allostatic measures in occupational health care, for instance.

\section{Declaration of conflicting interests}

The author(s) declared no potential conflicts of interest with respect to the research, authorship, and/or publication of this article.

\section{Funding}

The author(s) disclosed receipt of the following financial support for the research, authorship, and/or publication of this article: T.H. was supported by Ella and Georg Ehrnrooth Foundation. M.V. was supported by the Academy of Finland (grant 258598).

\section{References}

Aalto AM, Elovainio M, Kivimäki M, et al. (2012) The Beck Depression Inventory and General Health Questionnaire as measures of depression in the general population: A validation study using the Composite International Diagnostic Interview as the gold standard. Psychiatry Research 197(1-2): 163-171.

Aben I, Verhey F, Lousberg R, et al. (2002) Validity of the beck depression inventory, hospital anxiety and depression scale, SCL-90, and Hamilton Depression Rating Scale as screening instruments for depression in stroke patients. Psychosomatics 43(5): 386-393.

Ahola K and Hakanen J (2007) Job strain, burnout, and depressive symptoms: A prospective study among dentists. Journal of Affective Disorders 104(1-3): 103-110.

Ahola K, Honkonen T, Isometsä E, et al. (2005) The relationship between job-related burnout and depressive disorders-Results from the Finnish Health 2000 Study. Journal of Affective Disorders 88(1): 55-62.

Ahola K, Honkonen T, Isometsä E, et al. (2006a) Burnout in the general population. Results from the Finnish Health 2000 Study. Social Psychiatry and Psychiatric Epidemiology 41(1): 11-17.

Ahola K, Honkonen T, Kivimäki M, et al. (2006b) Contribution of burnout to the association between job strain and depression: The health 2000 study. Journal of Occupational and Environmental Medicine 48(10): 1023-1030.

Aikens JE, Reinecke MA, Pliskin NH, et al. (1999) Assessing depressive symptoms in multiple sclerosis: Is it necessary to omit items from the original Beck Depression Inventory? Journal of Behavioral Medicine 22(2): 127-142.

Aromaa A and Koskinen S (2004) Health and Functional Capacity in Finland. Baseline Results of the Health 2000 Health Examination Survey (Vol. B12/2004). Helsinki: National Public Health Institute. 
Bakker AB, Schaufeli WB, Demerouti E, et al. (2000) Using equity theory to examine the difference between burnout and depression. Anxiety, Stress and Coping 13(3): 247-268.

Beck AT, Steer RA and Garbin MG (1988) Psychometric properties of the Beck Depression Inventory: Twenty-five years of evaluation. Clinical Psychology Reviews 8: 77-100.

Bellingrath S, Weigl T and Kudielka BM (2009) Chronic work stress and exhaustion is associated with higher allostatic load in female school teachers. Stress 12(1): 37-48.

Bianchi R, Boffy C, Hingray C, et al. (2013) Comparative symptomatology of burnout and depression. Journal of Health Psychology 18(6): 782-787.

Cintra A, Bhatnagar M, Chadi G, et al. (1994) Glial and neuronal glucocorticoid receptor immunoreactive cell populations in developing, adult, and aging brain. Annals of New York Academic Sciences 746: 42-61.

Danhof-Pont MB, van Veen T and Zitman FG (2011) Biomarkers in burnout: A systematic review. Journal of Psychosomatic Research 70(6): 505-524.

Das S and O'Keefe JH (2006) Behavioral cardiology: Recognizing and addressing the profound impact of psychosocial stress on cardiovascular health. Current Atherosclerosis Reports 8(2): 111-118.

Everson SA, Kaplan GA, Goldberg DE, et al. (2000) Hypertension incidence is predicted by high levels of hopelessness in Finnish men. Hypertension 35(2): 561-567.

Garcia-Izquierdo M and Rios-Risquez MI (2012) The relationship between psychosocial job stress and burnout in emergency departments: An exploratory study. Nursing Outlook 60(5): 322-329.

Goldberg DP (1972) The Detection of Psychiatric Illness by Questionnaire. London: Oxford University Press.

Goldberg DP, Gater R, Sartorius N, et al. (1997) The validity of two versions of the GHQ in the WHO study of mental illness in general health care. Psychological Medicine 27(1): 191-197.

Goldberg DP and Williams P (1978) A User's Guide to the General Health Questionnaire. Windsor: NFER-Nelson.

Grewen K, Girdler SS, West SG, et al. (2000) Stable pessimistic attributions interact with socioeconomic status to influence blood pressure and vulnerability to hypertension. Journal of Women's Health and Gender Based Medicine 9(8): 905-915.

Heim C, Ehlert U and Hellhammer DH (2000) The potential role of hypocortisolism in the pathophysiology of stress-related bodily disorders. Psychoneuroendocrinology 25(1): 1-35.

Heistaro S (2008) Methodology Report. Health 2000 Survey. Helsinki: KTL - National Public Health Institute.

Honkonen T, Ahola K, Pertovaara M, et al. (2006) The association between burnout and physical illness in the general population-Results from the Finnish Health 2000 Study. Journal of Psychosomatic Research 61(1): 59-66.

Juster RP, McEwen BS and Lupien SJ (2010) Allostatic load biomarkers of chronic stress and impact on health and cognition. Neuroscience and Biobehavioral Review 35(1): 2-16.

Juster RP, Sindi S, Marin MF, et al. (2011a) A clinical allostatic load index is associated with burnout symptoms and hypocortisolemic profiles in healthy workers. Psychoneuroendocrinology 36(6): 797-805.

Juster RP, Marin MF, Sindi S, et al. (2011b) Allostatic load associations to acute, 3-year and 6-year prospective depressive symptoms in healthy older adults. Physiology and Behavior 104(2): 360-364.

Kalimo R, Hakanen J and Toppinen-Tanner S (2006) Maslachin yleinen työuupumuksen arviointimenetelmä MBI-GS [The Finnish version of Maslach's Burnout Inventory-General Survey]. Helsinki: Finnish Institute of Occupational Health.

Karapolat H, Demir E, Bozkaya YT, et al. (2009) Comparison of hospital-based versus homebased exercise training in patients with heart failure: Effects on functional capacity, quality of life, psychological symptoms, and hemodynamic parameters. Clinical Research in Cardiology 98(10): 635-642.

Knekt P, Lindfors O, Härkänen T, et al. (2008) Randomized trial on the effectiveness of long-and short-term psychodynamic psychotherapy and solution-focused therapy on psychiatric symptoms during a 3-year follow-up. Psychological Medicine 38(5): 689-703.

Langelaan S, Bakker AB, Schaufeli WB, et al. (2007) Is burnout related to allostatic load? International Journal of Behavioral Medicine 14(4): 213-221. 
Mäkikangas A, Feldt T, Kinnunen U, et al. (2006) The factor structure and factorial invariance of the 12-item General Health Questionnaire (GHQ12) across time: Evidence from two communitybased samples. Psychological Assessment 18(4): 444-451.

Maslach C, Jackson SE and Leiter MP (1996) Maslach Burnout Inventory Manual (3rd edn). Palo Alto, CA: Consulting Psychologist Press.

Maslach C, Schaufeli WB and Leiter MP (2001) Job burnout. Annual Review of Psychology 52: 397-422.

McCabe CJ, Thomas KJ, Brazier JE, et al. (1996) Measuring the mental health status of a population: A comparison of the GHQ-12 and the SF-36 (MHI-5). British Journal of Psychiatry 169(4): 516-521.

McEwen BS (1998) Stress, adaptation, and disease. Allostasis and allostatic load. Annals of New York Academic Sciences 840: 33-44.

McEwen BS (2003) Mood disorders and allostatic load. Biological Psychiatry 54(3): 200-207.

McEwen BS and Stellar E (1993) Stress and the individual. Mechanisms leading to disease. Archives of Internal Medicine 153(18): 2093-2101.

Mohren DC, Swaen GM, Kant IJ, et al. (2003) Common infections and the role of burnout in a Dutch working population. Journal of Psychosomatic Research 55(3): 201-208.

Mommersteeg PM, Heijnen CJ, Kavelaars A, et al. (2006) Immune and endocrine function in burnout syndrome. Psychosomatic Medicine 68(6): 879-886.

Nabi H, Kivimäki M, Suominen S, et al. (2010) Does depression predict coronary heart disease and cerebrovascular disease equally well? The Health and Social Support Prospective Cohort Study. International Journal of Epidemiology 39(4): 1016-1024.

Nuevo R, Dunn G, Dowrick C, et al. (2009) Crosscultural equivalence of the Beck Depression Inventory: A five-country analysis from the ODIN study. Journal of Affective Disorders 114(1-3): 156-162.

Penninkilampi-Kerola V, Miettunen J and Ebeling $\mathrm{H}$ (2006) A comparative assessment of the factor structures and psychometric properties of the GHQ-12 and the GHQ-20 based on data from a Finnish population-based sample. Scandinavian Journal of Psychology 47(5): 431-440.

Pruessner JC, Hellhammer DH and Kirschbaum C (1999) Low self-esteem, induced failure and the adrenocortical stress response. Personality and Individual Differences 27: 366-378.

Ranjit N, Diez-Roux AV, Shea S, et al. (2007) Psychosocial factors and inflammation in the multi-ethnic study of atherosclerosis. Archives of Internal Medicine 167(2): 174-181.

Roy B, Diez-Roux AV, Seeman T, et al. (2010) Association of optimism and pessimism with inflammation and hemostasis in the MultiEthnic Study of Atherosclerosis (MESA). Psychosomatic Medicine 72(2): 134-140.

Sandström A, Rhodin IN, Lundberg M, et al. (2005) Impaired cognitive performance in patients with chronic burnout syndrome. Biological Psychology 69(3): 271-279.

Sapolsky RM, Romero LM and Munck AU (2000) How do glucocorticoids influence stress responses? Integrating permissive, suppressive, stimulatory, and preparative actions. Endocrinology Reviews 21(1): 55-89.

Scarpa A and Luscher KA (2002) Self-esteem, cortisol reactivity, and depressed mood mediated by perceptions of control. Biological Psychology 59(2): 93-103.

Schaufeli W and Enzmann D (1998) The Burnout Companion to Study and Practice: A Critical Analysis. London: Taylor \& Francis.

Schaufeli W, Leiter M, Maslach C, et al. (1996) Maslach Burnout Inventory-General Survey (MBIGS). In: Maslach $\mathrm{C}$, Jackson $\mathrm{S}$ and Leiter M (eds) Maslach Burnout Inventory Manual (3rd edn). Palo Alto, CA: Consulting Psychologist Press, pp. 19-26.

Schmitz N, Kruse J and Tress W (1999) Psychometric properties of the General Health Questionnaire (GHQ-12) in a German primary care sample. Acta Psychiatrica Scandinavica 100(6): 462-468.

Schnorpfeil P, Noll A, Schulze R, et al. (2003) Allostatic load and work conditions. Social Science \& Medicine 57(4): 647-656.

Schutte N, Toppinen S, Kalimo R, et al. (2000) The factorial validity of the Maslach Burnout InventoryGeneral Survey (MBI-GS) across occupational groups and nations. Journal of Occupational and Organisational Psychology 73: 53-66.

Seeman TE, Singer BH, Rowe JW, et al. (1997) Price of adaptation-allostatic load and its health consequences. MacArthur studies of successful aging. Archives of Internal Medicine 157(19): 2259-2268.

Sheline YI, Barch DM, Donnelly JM, et al. (2001) Increased amygdala response to masked 
emotional faces in depressed subjects resolves with antidepressant treatment: An fMRI study. Biological Psychiatry 50(9): 651-658.

Shirom A (2003) Job-related burnout: A review. In: Quick JC and Tetrick LE (eds) Handbook of Occupational Health Psychology. Washington, DC: American Psychological Association, pp. 245-264.

Shirom A and Melamed S (2005) Does burnout affect physical health? A review of the evidence. In: Alexander-Stamatios AG and Cooper CL (eds) Research Companion to Organizational Health Psychology. Cheltenham: Edward Elgar Publishing, pp. 599-622.

Steptoe A, O'Donnell K, Badrick E, et al. (2008) Neuroendocrine and inflammatory factors associated with positive affect in healthy men and women: The Whitehall II study. American Journal of Epidemiology 167(1): 96-102.

Taris T, Schreurs P and Schaufeli W (1999) Construct validity of the Maslach Burnout InventoryGeneral Survey: Two sample examination of its factor structure and correlates. Work and Stress 13: 223-237.

Thayer JF, Smith M, Rossy LA, et al. (1998) Heart period variability and depressive symptoms: Gender differences. Biological Psychiatry 44(4): 304-306.

Toker S and Biron M (2012) Job burnout and depression: Unraveling their temporal relationship and considering the role of physical activity. Journal of Applied Psychology 97(3): 699-710.

Toker S, Melamed S, Berliner S, et al. (2012) Burnout and risk of coronary heart disease: A prospective study of 8838 employees. Psychosomatic Medicine 74(8): 840-847.

Warr P (1987) Work, Unemployment and Mental Health. Oxford: Clarendon Press.

World Health Organization (1992) The ICD-10 Classification of Mental and Behavioural Disorders: Clinical Descriptions and Diagnostic Guidelines. Geneva: World Health Organization. 